\title{
Fabrication of an Electrically Conductive Adhesive for Skin Mounted Bioelectronic Devices
}

\author{
Sunland L. Gong'; Min Ku Kim²; Tae Hoo Chang'; Chi Hwan Lee ${ }^{2}$ \\ ${ }^{1}$ Indiana University School of Medicine \\ ${ }^{2}$ Purdue University Weldon School of Biomedical Engineering
}

\section{Background}

Skin mounted bioelectronics are difficult to integrate with the skin since biocompatible adhesives are not conductive or unsuitable for long-term use. Skin conformability is essential but strong adhesives can damage soft tissue in younger and frail individuals as well as the device during removal. Developing a noninvasive long-lasting biocompatible conductive adhesive for skin that can be used with bioelectronics allows for better treatment options and the improvement of patient outcomes.

\section{Methods}

This study creates a soft hydrogel using graphene oxide flakes $(\mathrm{GO})$ and polyvinyl alcohol. Networked GO is reduced in a solution of sodium dithionite and sodium hydroxide to form a conductive network within the hydrogel. Adhesive properties are achieved by incorporating a polyacrylic acid polymer into the hydrogel with the addition of N-hydroxysulfosuccinimide (NHS) groups to the polymer. NHS reacts with amine groups found on tissue to form covalent bonds that can be released with a biocompatible trigger solution of sodium bicarbonate and glutathione.

\section{Results}

Hydration of the hydrogel at $65^{\circ} \mathrm{C}$ demonstrated that the hydrogel swelled anistropically with swelling ratios of 1.05/1.06/5.5 (length/width/thickness). This showed that the hydrogel can integrate into various surfaces without deformation. The hydrogel demonstrated an impedance of $106.1 \sim 164.6 \Omega \cdot \mathrm{m}(20 \sim 500 \mathrm{~Hz})$, which is comparable to conventional devices. The hydrogel was chemically bound to amine functionalized polydimethylsiloxane (PDMS) and glass. Peel test showed peak adhesion forces of $100.5 \mathrm{~N} \cdot \mathrm{m}^{-1}$ (Force.Width ${ }^{-1}$ ) when bound to PDMS or glass. Signal quality of the hydrogel showed that the hydrogels demonstrated ECG and EMG signals comparable to commercially available materials.

\section{Conclusions}

The importance of this study is to create a soft material that bonds between electrodes and skin. The results demonstrate that the hydrogel has electrical characteristics comparable to conventional electrodes for use in ECG and EMG. In addition, it can create adhesion via chemical bonds that can be released on demand. 